Molecules 2004, 9, 204-212

molecules

ISSN 1420-3049

http://www.mdpi.org

\title{
Synthesis of Some New 4,5-Substituted-4H-1,2,4-triazole-3-thiol Derivatives
}

\author{
A. Cansız, M. Koparır * and A. Demirdağ

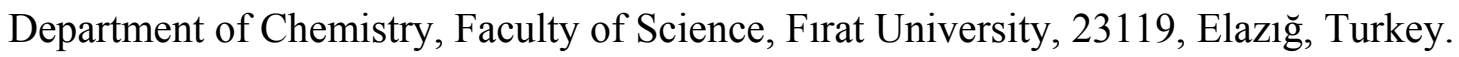

*Author to whom correspondence should be addressed; e-mail: $\underline{\text { mkoparir@,hotmail.com }}$

Received: 11 July 2003; in revised form: 9 February 2004 / Accepted: 2 March 2004 / Published: 31 March 2004

\begin{abstract}
In this study appropriate hydrazide compounds, furan-2-carboxylic acid hydrazide (1) and phenylacetic acid hydrazide (2) were converted into 1,4-substituted thiosemicarbazides 4a-e and 5a-e and 4-amino-5-(furan-2-yl or benzyl)-4H-1,2,4-triazole3-thiols 7 and 10. The 1,4-substituted thiosemicarbazides were then converted into 5-(furan-2-yl or benzyl)-4-(aryl)-4H-1,2,4-triazole-3-thiols 8a-e and 9a-e. In addition, the azomethines 11a-d and 12a-d were prepared from the corresponding arylaldehydes and the 4-amino-5-(furan-2-yl or benzyl)-4H-1,2,4-triazole-3-thiols 7 and 10. The structures of all the synthesized compounds were confirmed by elemental analyses, IR, ${ }^{1} \mathrm{H}-\mathrm{NMR}$ and ${ }^{13} \mathrm{C}-\mathrm{NMR}$ spectra.
\end{abstract}

Keywords: 4H-1,2,4-triazole-3-thiols; azomethines; thiosemicarbazides.

\section{Introduction}

Derivatives of 1,2,4-triazole are known to exhibit anti-inflammatory [1,2], antiviral [3], analgesic [4], antimicrobial [5-7], anticonvulsant [8] and antidepressant activity [9], the latter being usually explored by the forced swim test [10,11]. Among the pharmacological profiles of 1,2,4-triazoles, their antimicrobial, anticonvulsant and antidepressant properties seem to be the best documented. New changing problems in plant protection technology have promoted research to discover more efficient pesticides. In particular the development of herbicides, now an unavoidable means to selectively control 
the growth of weeds, resulted in a whole range of azoles exhibiting high levels of activity, application flexibility, crop tolerance and low levels of toxicity to mammals. Triazoles play an important role among this class of heterocycles. A series of 1,2,4-triazole derivatives [11] have been patented and extensively employed in agriculture. We now report the synthesis of 20 original compounds derived from furan-2-carboxylic acid hydrazide and phenylacetic hydrazide with the purpose of investigating in the future their possible antibacterial and antifungal activity.

\section{Results and Discussion}

The new derivatives were prepared following the reaction sequences depicted in Scheme 1. Initial compounds were prepared from available furan-2-carboxylic acid hydrazide (1), and phenylacetic acid hydrazide (2). Potassium 3-(2-furoyl) or (phenylacetyl) dithiocarbazates 3, 6 were prepared by reaction of compounds 1 and $\mathbf{2}$ with carbon disulfide in ethanolic potassium hydroxide. 1-(2-Furoyl or phenylacetyl)-4-substituted thiosemicarbazides 4a-e and 5a-e were prepared in yields ranging from 88 to $95 \%$ by the condensation of $\mathbf{1}$ and $\mathbf{2}$ with arylisothiocyanates. Ring closure of arylthiosemicarbazides in an alkaline medium is a well known method for the synthesis of 1,2,4-triazoles, and 5-(furan-2-yl or benzyl)-4-(aryl)-4H-1,2,4-triazole-3-thiols 8a-e and 9a-e were obtained in 62-79\% yields from the respective 4a-e, 5a-e by this method. Compounds 7 and $\mathbf{1 0}$ were obtained from the reaction of 3 and 6 with hydrazide hydrate under reflux in solution. When an arylaldehyde was added to $\mathbf{7}$ or $\mathbf{1 0}$ in ethanol, the reactions gave $\mathbf{1 1 a - d ,} \mathbf{1 2 a - d}$.

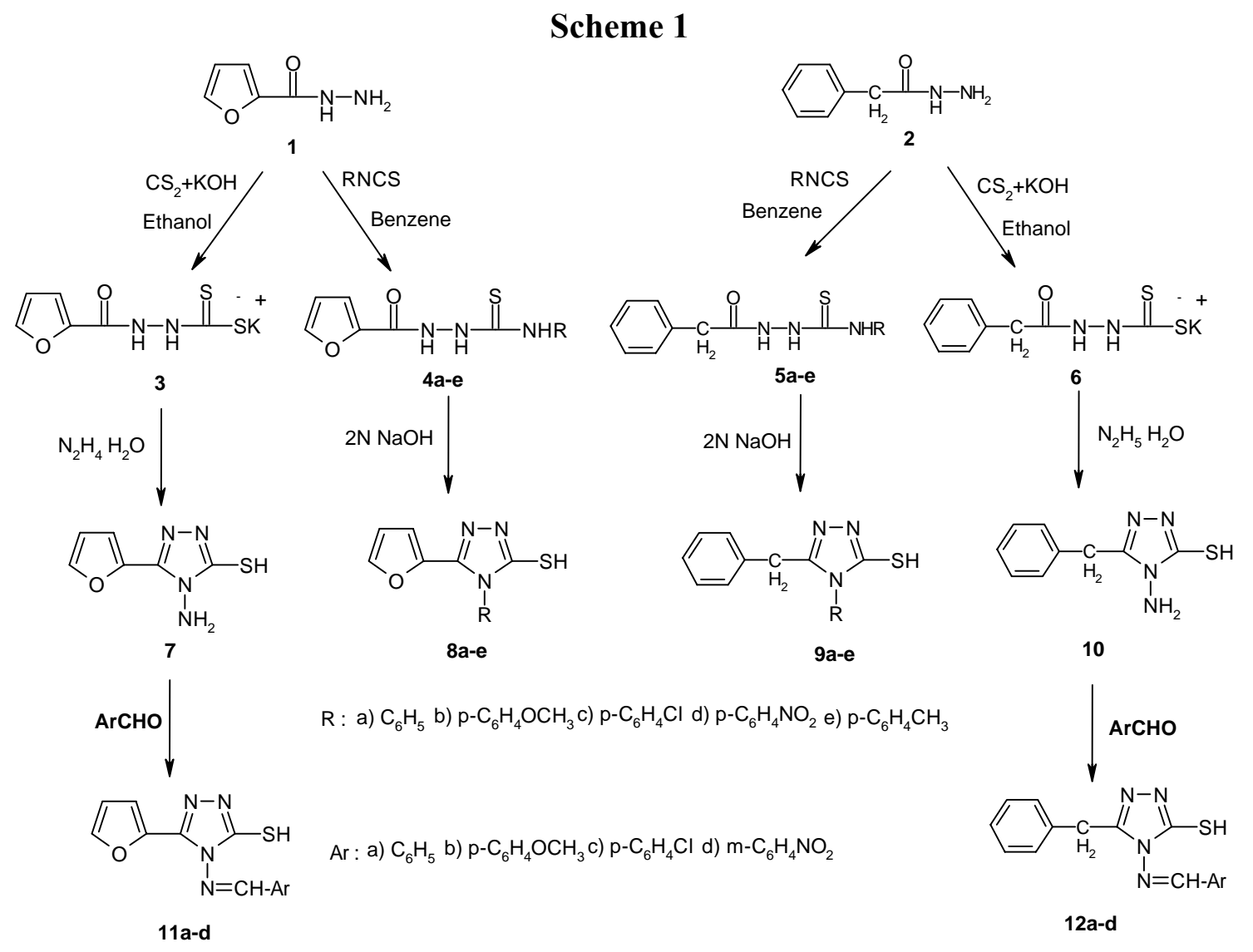


The IR spectra of the 1,4-substituted-thiosemicarbazide derivatives 4a-e, 5a-e have $\mathrm{C}=\mathrm{O}$ stretching bands at $1687-1672 \mathrm{~cm}^{-1}$ and $\mathrm{C}=\mathrm{S}$ stretching bands at $1290-1250 \mathrm{~cm}^{-1}$. The N-H protons of 4a-e, 5a-e were observed at 9.20-10.15 ppm, $(\mathrm{O}=\mathrm{C}-\mathrm{NH}-\mathrm{NH}-\mathrm{C}=\mathrm{S})$ and 8.00-8.25 ppm $(\mathrm{S}=\mathrm{C}-\mathrm{NH}-\mathrm{Ar})$. Compounds 7, 8a-e, 9a-e, 10, 11a-d, 12a-d exist as thiol-thione tautomers as indicated by their IR spectra which showed a band due to $\mathrm{SH}$ and four bands due to $\mathrm{N}-\mathrm{C}=\mathrm{S}$ I, II, III, IV. The azomethine derivatives 11a-d, 12a-d were charcterized by the presence of the methine protons $(\mathrm{N}=\mathrm{CH})$ at $9.18-10.33 \mathrm{ppm}$. The data of all the compounds are given in the Experimental section.

\section{Experimental}

\section{General}

Melting points were determined in open capillary tubes on a digital Gallenkamp melting point apparatus and are uncorrected. The IR spectra were recorded for $\mathrm{KBr}$ disks with a Mattson $1000 \mathrm{FT}$-IR spectrometer. ${ }^{1} \mathrm{H}-\mathrm{NMR}$ spectra were recorded on a FX 90 JEOL $90 \mathrm{MHz} \mathrm{NMR}$, spectrometer in $\mathrm{CDCl}_{3}+$ DMSO-d $_{6}$ with TMS as an internal standard. Elemental analyses were done on a LECO-CHNS-938. Starting materials were obtained from Fluka or Aldrich.

General Procedure for Preparation of Potassium 3-(2-furoyl) or (phenylacetyl)dithiocarbazates 3 and 6.

Carbon disulfide $(0.15$ mole $)$ was added to a solution of potassium hydroxide $(0.15$ mole $)$, absolute ethanol $(200 \mathrm{~mL})$ and the appropriate furan-2-carboxylic acid hydrazide (1) or phenylacetic acid hydrazide (2) (0.10 mole). This mixture was diluted with absolute ethanol $(150 \mathrm{~mL})$ and agitated for 12-18 hours. It was then diluted with dry ether $(250 \mathrm{~mL})$ and the products were filtered off and vacuum dried at $65{ }^{\circ} \mathrm{C}$. The salts prepared as described above were obtained in nearly quantitative yields and were used without further purification.

Potassium-3-(2-furoyl)dithiocarbazate (3): m.p. 222-225 ${ }^{\circ} \mathrm{C} ; \mathrm{IR} v\left(\mathrm{~cm}^{-1}\right): 3450,3310(\mathrm{~N}-\mathrm{H}), 1639$ $(\mathrm{C}=\mathrm{O}), 1270(\mathrm{C}=\mathrm{S}), 1248(\mathrm{C}-\mathrm{O}-\mathrm{C})$.

Potassium-3-(Phenylacetyl)dithiocarbazate (6): m.p. 212-255 ${ }^{\circ} \mathrm{C}$; IR v ( $\left.\mathrm{cm}^{-1}\right): 3375,3225(\mathrm{~N}-\mathrm{H}), 1670$ $(\mathrm{C}=\mathrm{O}), 1275(\mathrm{C}=\mathrm{S}), 1250(\mathrm{C}-\mathrm{O}-\mathrm{C})$.

General procedure for Preparation of 4a-e, 5a-e.

A mixture of 1 (or 2) (0.01 mole) and the appropriate aryl isothiocyanate $\left(0.01\right.$ mole) in dry $\mathrm{C}_{6} \mathrm{H}_{6}$ was refluxed for 6 hours. The solid material obtained on cooling was filtered off and recrystallized from methanol. 
1-(2-furoyl)-4-Phenylthiosemicarbazide (4a): From phenylisothiocyanate, yield 92\%; m.p. 184-185 ${ }^{\circ} \mathrm{C}$; IR v $\left(\mathrm{cm}^{-1}\right)$ : 3365, $3315(\mathrm{~N}-\mathrm{H}), 1672(\mathrm{C}=\mathrm{O}), 1274(\mathrm{C}=\mathrm{S}), 1251$ (C-O-C); ${ }^{1} \mathrm{H}-\mathrm{NMR} \delta$ (ppm): 6.68-7.20 (m, 3H, furan), 7.42-7.92 (m, 5H, Ar-H), 8.11-8.19 (br, 1H, -NH-Ar), 9.20-9.92 (br, 2H, 2xNH). Calcd. for $\mathrm{C}_{12} \mathrm{H}_{11} \mathrm{~N}_{3} \mathrm{O}_{2} \mathrm{~S}(261)$; (\%): C, 55.16; $\mathrm{H}, 4.24 ; \mathrm{N}, 16.08 ; \mathrm{S}, 12.27$; found (\%): $\mathrm{C}, 55.27 ; \mathrm{H}, 4.11, \mathrm{~N}$, 15.97; S, 12.13.

1-(2-furoyl)-4-(4-methoxyphenyl)thiosemicarbazide (4b): From p-methoxphenylisothiocyanate, yield 93\%; m.p. 162-163 ${ }^{\circ} \mathrm{C}$; IR v $\left(\mathrm{cm}^{-1}\right)$ : 3355, $3320(\mathrm{~N}-\mathrm{H}), 1678(\mathrm{C}=\mathrm{O}), 1271$ (C=S), 1253 (C-O-C); ${ }^{1} \mathrm{H}-\mathrm{NMR} \delta$ (ppm): 3.79 (s, 3H, -O-CH 3 ) 5.90-6.20 (m, 3H, furan), 6.55-6.90 (m, 4H, Ar-H), 8.21-8.25 (br, 1H, -NH-Ar), 9.21-9.92 (br, 2H, 2xNH). Calcd. for $\mathrm{C}_{13} \mathrm{H}_{13} \mathrm{~N}_{3} \mathrm{O}_{3} \mathrm{~S}$ (291); (\%): C, 53.60; H, 4.50; N, 14.42; S, 11.01; found (\%): C, 53.45; H, 4.51, N, 14.47; S, 10.98 .

1-(2-furoyl)-4-(4-chlorophenyl)thiosemicarbazide (4c): From p-chlorophenylisothiocyanate, yield 90\%; m.p. 188-189 ${ }^{\circ} \mathrm{C}$; IR v $\left(\mathrm{cm}^{-1}\right)$ : 3354, $3321(\mathrm{~N}-\mathrm{H}), 1673(\mathrm{C}=\mathrm{O}), 1268(\mathrm{C}=\mathrm{S}), 1249(\mathrm{C}-\mathrm{O}-\mathrm{C}) ;{ }^{1} \mathrm{H}-\mathrm{NMR} \delta$ (ppm): 6.67-7.21 (m, 3H, furan), 7.22-7.90 (m, 4H, Ar-H), 8.18-8.20 (br, 1H, -NH-Ar), 9.71-10.02 (br, $2 \mathrm{H}, 2 \times N H)$. Calcd. for $\mathrm{C}_{12} \mathrm{H}_{10} \mathrm{ClN}_{3} \mathrm{O}_{2} \mathrm{~S}(295)$; (\%): C, 48.74; H, 3.41; N, 14.21; S, 10.84; found (\%): C, $48.45 ; \mathrm{H}, 3.43, \mathrm{~N}, 14.17$; S, 10.88 .

1-(2-furoyl)-4-(4-nitrophenyl)thiosemicarbazide (4d): From p-nitrophenylisothiocyanate, yield 95\%; m.p. 210-211 ${ }^{\circ} \mathrm{C}$; IR v (cm $\left.{ }^{-1}\right)$ : 3344, $3311(\mathrm{~N}-\mathrm{H}), 1422\left(\mathrm{NO}_{2}\right), 1679(\mathrm{C}=\mathrm{O}), 1274(\mathrm{C}=\mathrm{S}), 1254(\mathrm{C}-\mathrm{O}-\mathrm{C})$; ${ }^{1} \mathrm{H}-\mathrm{NMR} \delta$ (ppm): 6.70-7.28 (m, 3H, furan), 7.56-7.76 (m, 4H, Ar-H), 8.00-8.14 (br, 1H, -NH-Ar), 9.21-10.11 (br, 2H, 2xNH). Calcd. for $\mathrm{C}_{12} \mathrm{H}_{10} \mathrm{~N}_{4} \mathrm{O}_{4} \mathrm{~S}$ (306); (\%): C, 47.06; H, 3.29; N, 18.29; S, 10.47; found (\%): C, 47.10; H, 3.23, N, 18.27; S, 10.40 .

1-(2-furoyl)-4-(4-methylphenyl)thiosemicarbazide (4e): From p-methylphenylisothiocyanate, yield 91\%; m.p. 165-167 ${ }^{\circ} \mathrm{C}$; IR v $\left(\mathrm{cm}^{-1}\right): 3449,3321(\mathrm{~N}-\mathrm{H}), 1683(\mathrm{C}=\mathrm{O}), 1269(\mathrm{C}=\mathrm{S}), 1241(\mathrm{C}-\mathrm{O}-\mathrm{C})$; ${ }^{1} \mathrm{H}-\mathrm{NMR} \delta$ (ppm): 2.28 (s, 3H, $\mathrm{CH}_{3}$ ), 6.71-7.26 (m, 3H, furan), 7.54-7.72 (m, 4H, Ar-H), 8.20-8.25 (br, 1H, -NH-Ar), 9.97-10.15 (br, 2H, 2xNH). Calcd. for $\mathrm{C}_{13} \mathrm{H}_{13} \mathrm{~N}_{3} \mathrm{O}_{2} \mathrm{~S}$ (275); (\%): C, 56.71; H, 4.76; N, 15.26; S, 11.65; found (\%): C, 56.54; H, 4.71, N, 14.97; S, 11.64 .

1-Phenylacetyl-4-phenylhiosemicarbazide (5a): From phenylisothiocyanate, yield 94\%; m.p. 160-161 ${ }^{\circ} \mathrm{C}$; IR v $\left(\mathrm{cm}^{-1}\right):$ 3450, $3310(\mathrm{~N}-\mathrm{H}), 1687(\mathrm{C}=\mathrm{O}), 1290(\mathrm{C}=\mathrm{S}) ;{ }^{1} \mathrm{H}-\mathrm{NMR} \delta(\mathrm{ppm}): 3.35\left(\mathrm{~s}, 2 \mathrm{H}, \mathrm{CH}_{2}\right)$, 7.30-7.44 (m, 10H, Ar-H), 8.21-8.23 (br, 1H, -NH-Ar), 9.95-10.11 (br, 2H, 2xNH). Calcd. for $\mathrm{C}_{15} \mathrm{H}_{15} \mathrm{~N}_{3} \mathrm{OS}(285) ;(\%): \mathrm{C}, 63.13 ; \mathrm{H}, 5.30 ; \mathrm{N}, 14.72 ; \mathrm{S}, 11.24$; found (\%): C, 63.09; H, 5.35, N, 14.67; S, 11.19.

1-Phenylacetyl-4-(4-methoxyphenyl)thiosemicarbazide (5b): From p-methoxphenylisothiocyanate, yield 92\%; m.p. 157-159 ${ }^{\circ} \mathrm{C}$; v $\left(\mathrm{cm}^{-1}\right)$ : 3452, $3312(\mathrm{~N}-\mathrm{H}), 1686(\mathrm{C}=\mathrm{O}), 1274(\mathrm{C}=\mathrm{S})$; ${ }^{1} \mathrm{H}-\mathrm{NMR} \delta(\mathrm{ppm})$ : $3.39\left(\mathrm{~s}, 2 \mathrm{H}, \mathrm{CH}_{2}\right), 4.25$ (s, 3H, $\left.-\mathrm{O}-\mathrm{CH}_{3}\right), 6.77-7.30$ (m, 9H, Ar-H), 8.11-8.13 (br, 1H, -NH-Ar), 
9.98-10.13 (br, 2H, 2xNH). Calcd. for $\mathrm{C}_{16} \mathrm{H}_{17} \mathrm{~N}_{3} \mathrm{O}_{2} \mathrm{~S}$ (315); (\%): C, 60.93; H, 5.43; N, 13.32; S, 10.47; found (\%): C, 61.01; H, 5.25, N, 13.32; S, 10.49 .

1-Phenylacetyl-4-(4-chlorophenyl)thiosemicarbazide (5c): From p-chlorophenylisothiocyanate, yield 90\%; m.p. 164-165 ${ }^{\circ} \mathrm{C}$; IR $v\left(\mathrm{~cm}^{-1}\right): 3482,3317(\mathrm{~N}-\mathrm{H}), 1675(\mathrm{C}=\mathrm{O}), 1267(\mathrm{C}=\mathrm{S}) ;{ }^{1} \mathrm{H}-\mathrm{NMR} \delta(\mathrm{ppm}): 3.40$ (s, 2H, $\mathrm{CH}_{2}$ ), 7.31-7.44 (m, 9H, Ar-H), 8.13-8.17 (br, 1H, -NH-Ar), 10.11-10.13 (br, 2H, 2xNH). Calcd. for $\mathrm{C}_{15} \mathrm{H}_{14} \mathrm{ClN}_{3} \mathrm{OS}$ (319); (\%): C, 56.33; H, 4.41; N, 13.14; S, 10.03; found (\%): C, 56.21; H, 4.39, N, $13.12 ; \mathrm{S}, 9.99$.

1-Phenylacetyl-4-(4-nitrophenyl)thiosemicarbazide (5d): From p-nitrophenylisothiocyanate, yield 93\%; m.p. 187-188 ${ }^{\circ} \mathrm{C}$; IR v $\left(\mathrm{cm}^{-1}\right): 3451,3322(\mathrm{~N}-\mathrm{H}), 1672(\mathrm{C}=\mathrm{O}), 1266(\mathrm{C}=\mathrm{S}) ;{ }^{1} \mathrm{H}-\mathrm{NMR} \delta(\mathrm{ppm}): 3.74(\mathrm{~s}$, 2H, $\mathrm{CH}_{2}$ ), 7.37-7.87 (m, 9H, Ar-H), 8.19-8.22 (br, 1H, -NH-Ar), 10.13-10.15 (br, 2H, 2xNH). Calcd. for $\mathrm{C}_{15} \mathrm{H}_{14} \mathrm{~N}_{4} \mathrm{O}_{3} \mathrm{~S}$ (330); (\%): C, 54.54; H, 4.27; N, 16.96; S, 9.71; found (\%): C, 54.41; H, 4.29, N, 17.01; S, 9.69 .

1-Phenylacetyl-4-(4-methylphenyl)thiosemicarbazide (5e): From p-methylphenylisothiocyanate, yield 88\%; m.p. 160-161 ${ }^{\circ} \mathrm{C}$; IR $v\left(\mathrm{~cm}^{-1}\right): 3450,3329(\mathrm{~N}-\mathrm{H}), 1678(\mathrm{C}=\mathrm{O}), 1250(\mathrm{C}=\mathrm{S}) ;{ }^{1} \mathrm{H}-\mathrm{NMR} \delta(\mathrm{ppm}): 2.28$ (s, 3H, $\mathrm{CH}_{3}$ ), 3.53 (s, 2H, CH${ }_{2}$ ), 7.30-7.45 (m, 9H, Ar-H), 8.17-8.24 (br, 1H, -NH-Ar), 10.13-10.15 (br, $2 \mathrm{H}, 2 \mathrm{xNH})$. Calcd. for $\mathrm{C}_{16} \mathrm{H}_{17} \mathrm{~N}_{3} \mathrm{OS}(299)$; (\%): C, 64.19; H, 5.72; N, 14.03; S, 10.71; found (\%): $\mathrm{C}$, $64.11 ; \mathrm{H}, .69, \mathrm{~N}, 14.01 ; \mathrm{S}, 10.59$.

\section{General Procedure for the Preparation of Compounds $\mathbf{7}$ and $\mathbf{1 0 .}$}

A suspension of the potassium salt 3 (or 6 ) (20 mmoles), $95 \%$ hydrazine ( 40 mmoles) and water (2 $\mathrm{mL}$ ) was refluxed with stirring for 0.5 to 1 hours. The color of the reaction mixture changed to green, hydrogen sulfide was evolved and a homogeneous solution resulted. A white solid was precipitated by dilution with cold water $(100 \mathrm{~mL})$ and acidification with concentrated hydrochloric acid. This product was filtered, washed with cold water $(2 \times 30 \mathrm{~mL})$ and recrystallized from ethanol or ethanol-water.

4-Amino-5-furan-2-yl-4H-1,2,4-triazole-3-thiol (7): Yield 45\%; m.p. 202-203 ${ }^{\circ} \mathrm{C}$; IR v $\left(\mathrm{cm}^{-1}\right)$ : 3329 $(\mathrm{N}-\mathrm{H}), 2775(\mathrm{SH}), 1618(\mathrm{C}=\mathrm{N}), 1238$ (C-O-C), 1533, 1262, 1051, 952 (N-C=S, amide I, II, III and IV bands); ${ }^{1} \mathrm{H}-\mathrm{NMR} \delta$ (ppm): 5.80 (s, $2 \mathrm{H}, \mathrm{NH}_{2}$ ), 6.70-7.91 (m, $3 \mathrm{H}$, furan), 13.89 (s, $1 \mathrm{H}, \mathrm{SH}$ ); ${ }^{13} \mathrm{C}-\mathrm{NMR} \delta$ : 111.89, 11398, 139.90, 142.83, 145.20, 156.54. Calcd. for $\mathrm{C}_{6} \mathrm{H}_{6} \mathrm{~N}_{4} \mathrm{OS}$ (182); (\%): C, 39.55; H, 3.32; $\mathrm{N}$, 30.75 ; S, 17.60; found (\%): C, 39.49; H, 3.39, N, 31.01; S, 17.59 .

4-Amino-5-benzyl-4H-1,2,4-triazole-3-thiol (10): Yield 53\%; m.p. 207-209 ${ }^{\circ} \mathrm{C}$; IR v ( $\left.\mathrm{cm}^{-1}\right): 3337(\mathrm{~N}-\mathrm{H})$, $2350(\mathrm{SH}), 1620(\mathrm{C}=\mathrm{N}), 1535,1260,1050,950\left(\mathrm{~N}-\mathrm{C}=\mathrm{S}\right.$, amide I, II, III and IV bands); ${ }^{1} \mathrm{H}-\mathrm{NMR} \delta(\mathrm{ppm})$ : $5.01\left(\mathrm{~s}, 2 \mathrm{H}, \mathrm{NH}_{2}\right), 5.41\left(\mathrm{~s}, 2 \mathrm{H}, \mathrm{CH}_{2}\right), 6.72-7.02(\mathrm{~m}, 5 \mathrm{H}, \mathrm{Ar}-\mathrm{H}), 13.01(\mathrm{~s}, 1 \mathrm{H}, \mathrm{SH}) ;{ }^{13} \mathrm{C}-\mathrm{NMR} \delta: 35.41$, 
$111.89,113.98,125.71,127.91,128.31,139.90,141.54,142.83,145.20,156.54$. Calcd. for $\mathrm{C}_{9} \mathrm{H}_{10} \mathrm{~N}_{4} \mathrm{~S}$ (206); (\%): C, 52.41; H, 4.89; N, 27.16; S, 15.54; found (\%): C, 52.49; H, 4.87, N, 27.25.01; S, 15.59.

General Procedure for the Preparation of Compounds 8a-e and 9a-e.

A stirring mixture of compound $\mathbf{4 a}$ (or $\mathbf{4 b}-\mathbf{b}, \mathbf{5 a}-\mathbf{e})(1 \mathrm{mmole})$ and sodium hydroxide (40 $\mathrm{mg}, 1$ mmole, as a $2 \mathrm{~N}$ solution) was refluxed for 4 hours. After cooling, the solution was acidified with hydrochloric acid and the precipitate was filtered. The precipitate was then crystallized from ethanol.

5-Furan-2-yl-4-phenyl-4H-1,2,4-triazole-3-thiol (8a): Yield 68\%; m. p. 210-212 ${ }^{\circ} \mathrm{C}$; IR v $\left(\mathrm{cm}^{-1}\right)$ : 3321, $3250(\mathrm{~N}-\mathrm{H}), 2575(\mathrm{SH}), 1604(\mathrm{C}=\mathrm{N}), 1249$ (C-O-C), 1535, 1260, 1054, 948 (N-C=S, amide I, II, III and IV bands); ${ }^{1} \mathrm{H}-\mathrm{NMR} \delta$ (ppm): 5.97-6.34 (m, 3H, furan), 7.45-7.55 (m, 5H, Ar-H), 13.04 (s, 1H, SH). Calcd. for $\mathrm{C}_{12} \mathrm{H}_{9} \mathrm{~N}_{3} \mathrm{OS}(243)$; (\%): C, 59.24; H, 3.73; N, 17.27; S, 13.18; found (\%): C, 58.99; H, 3.69, N, $17.25 ; \mathrm{S}, 13.19$.

5-Furan-2-yl-4-(4-methoxyphenyl)-4H-1,2,4-triazole-3-thiol (8b): Yield 78\%; m. p. 245-246 ${ }^{\circ} \mathrm{C}$; IR v $\left(\mathrm{cm}^{-1}\right):$ 3331, $3258(\mathrm{~N}-\mathrm{H}), 2564(\mathrm{SH}), 1618(\mathrm{C}=\mathrm{N}), 1250(\mathrm{C}-\mathrm{O}-\mathrm{C}), 1538,1259,1048,948(\mathrm{~N}-\mathrm{C}=\mathrm{S}$, amide I, II, III and IV bands); ${ }^{1} \mathrm{H}-\mathrm{NMR} \delta$ (ppm): 3.88 (s, 3H, $\mathrm{OCH}_{3}$ ), 5.95-6.38 (m, 3H, furan), 7.09-7.55 (m, 4H, Ar-H), 14.00 (s, 1H, SH). Calcd. for $\mathrm{C}_{13} \mathrm{H}_{11} \mathrm{~N}_{3} \mathrm{O}_{2} \mathrm{~S}$ (273); (\%): C, 57.13; H, 4.06; N, 15.37; S, 11.73; found (\%): C, 57.19; H, 3.99, N, 15.25; S, 11.79 .

4-(4-Chlorophenyl-)5-Furan-2-yl)-4H-1,2,4-triazole-3-thiol (8c): Yield 72\%; m. p. 264-265 ${ }^{\circ} \mathrm{C}$; IR v $\left(\mathrm{cm}^{-1}\right):$ 3351, $3288(\mathrm{~N}-\mathrm{H}), 2564(\mathrm{SH}), 1625(\mathrm{C}=\mathrm{N}), 1250(\mathrm{C}-\mathrm{O}-\mathrm{C}), 1535,1260,1050,950(\mathrm{~N}-\mathrm{C}=\mathrm{S}$, amide I, II, III and IV bands); ${ }^{1} \mathrm{H}-\mathrm{NMR} \delta$ (ppm): 6.16-6.50 (m, 3H, furan), 7.45-7.78 (m, 4H, Ar-H), 13.98 (s, $1 \mathrm{H}, \mathrm{SH})$. Calcd. for $\mathrm{C}_{12} \mathrm{H}_{8} \mathrm{ClN}_{3} \mathrm{OS}(273)$; (\%): C, 51.90; H, 2.90; N, 15.13; S, 11.54; found (\%): C, 51.88; H, 2.99, N, 15.15; S, 11.49 .

4-(4-Nitrophenyl-)5-Furan-2-yl)-4H-1,2,4-triazole-3-thiol (8d): Yield 62\%; m. p. 187-188 ${ }^{\circ} \mathrm{C}$; IR v $\left(\mathrm{cm}^{-1}\right)$ : 3341, $3280(\mathrm{~N}-\mathrm{H}), 2580(\mathrm{SH}), 1625(\mathrm{C}=\mathrm{N}), 1250(\mathrm{C}-\mathrm{O}-\mathrm{C}), 1528\left(\mathrm{NO}_{2}\right), 1537,1263,1057,952$ (N-C=S, amide I, II, III and IV bands); ${ }^{1} \mathrm{H}-\mathrm{NMR} \delta$ (ppm): 5.91-6.95 (m, 3H, furan), 7.51-8.08 (m, 4H, Ar-H), 12.98 (s, 1H, SH). Calcd. for $\mathrm{C}_{12} \mathrm{H}_{8} \mathrm{~N}_{4} \mathrm{O}_{3} \mathrm{~S}$ (288); (\%): C, 50.00; H, 2.80; N, 19.43; S, 11.12; found (\%): C, 50.08; H, 2.89, N, 19.50; S, 11.15 .

5-Furan-2-yl-4-(4-methylphenyl)-4H-1,2,4-triazole-3-thiol (8e): Yield 79\%; m. p. 257-258 ${ }^{\circ} \mathrm{C}$; IR v $\left(\mathrm{cm}^{-1}\right)$ : 3351, $3285(\mathrm{~N}-\mathrm{H}), 2576(\mathrm{SH}), 1621(\mathrm{C}=\mathrm{N}), 1250(\mathrm{C}-\mathrm{O}-\mathrm{C}), 1534,1258,1050,951(\mathrm{~N}-\mathrm{C}=\mathrm{S}$, amide I, II, III and IV bands); ${ }^{1} \mathrm{H}-\mathrm{NMR} \delta$ (ppm): 2.46 (s, 3H, $\mathrm{CH}_{3}$ ), 5.94-6.36 (m, 3H, furan), 7.31-7.47 (m, 4H, Ar-H), 14.01 (s, 1H, SH). Calcd. for $\mathrm{C}_{13} \mathrm{H}_{11} \mathrm{~N}_{3} \mathrm{OS}$ (257); (\%): C, 60.68; H, 4.31; N, 16.33; S, 12.46; found (\%): C, 60.65; H, 4.39, N, 16.50; S, 12.42 . 
5-Benzyl-4-phenyl-4H-1,2,4-triazole-3-thiol (9a): Yield 73\%; m. p. 197-198 ${ }^{\circ} \mathrm{C}$; IR v ( $\left.\mathrm{cm}^{-1}\right): 3344,3282$ $(\mathrm{N}-\mathrm{H}), 2548(\mathrm{SH}), 1620(\mathrm{C}=\mathrm{N}), 1535,1263,1050,951\left(\mathrm{~N}-\mathrm{C}=\mathrm{S}\right.$, amide I, II, III and IV bands); ${ }^{1} \mathrm{H}-\mathrm{NMR}$ $\delta$ (ppm): $3.81\left(\mathrm{~s}, 2 \mathrm{H}, \mathrm{CH}_{2}\right), 7.10-7.45$ (m, 10H, Ar-H), 13.81 (s, 1H, SH). Calcd. for $\mathrm{C}_{15} \mathrm{H}_{13} \mathrm{~N}_{3} \mathrm{~S}(267)$; (\%): C, 67.39; H, 4.90; N, 15.72; S, 11.99; found (\%): C, 67.38; H, 4.87, N, 15.65; S, 12.09 .

5-Benzyl-4-(4-methoxyphenyl)-4H-1,2,4-triazole-3-thiol (9b): Yield 79\%; m.p. 177-178 ${ }^{\circ} \mathrm{C}$; IR v ( $\left.\mathrm{cm}^{-1}\right)$ : 3344, $3282(\mathrm{~N}-\mathrm{H}), 2570(\mathrm{SH}), 1606(\mathrm{C}=\mathrm{N}), 1538,1260,1050,950(\mathrm{~N}-\mathrm{C}=\mathrm{S}$, amide I, II, III and IV bands); ${ }^{1} \mathrm{H}-\mathrm{NMR} \delta(\mathrm{ppm}): 3.80\left(\mathrm{~s}, 2 \mathrm{H}, \mathrm{CH}_{2}\right), 3.88\left(\mathrm{~s}, 3 \mathrm{H}, \mathrm{OCH}_{3}\right), 6.95-7.25(\mathrm{~m}, 9 \mathrm{H}, \mathrm{Ar}-\mathrm{H}), 13.06(\mathrm{~s}, 1 \mathrm{H}, \mathrm{SH})$. Calcd. for $\mathrm{C}_{16} \mathrm{H}_{15} \mathrm{~N}_{3} \mathrm{OS}(297)$; (\%): C, 64.62; H, 5.08; N, 14.13; S, 10.78; found (\%): C, 64.58; H, 4.97, N, 14.09; S, 10.72 .

5-Benzyl-4-(4-chlorophenyl)-4H-1,2,4-triazole-3-thiol (9c): Yield 73\%; m.p. 187-188 ${ }^{\circ} \mathrm{C}$; IR v $\left(\mathrm{cm}^{-1}\right)$ : 3354, $3292(\mathrm{~N}-\mathrm{H}), 2580(\mathrm{SH}), 1606(\mathrm{C}=\mathrm{N}), 1535,1260,1050,950(\mathrm{~N}-\mathrm{C}=\mathrm{S}$, amide I, II, III and IV bands); ${ }^{1} \mathrm{H}-\mathrm{NMR} \delta: 3.84$ (s, 2H, CH 2$), 6.94-7.42(\mathrm{~m}, 9 \mathrm{H}, \mathrm{Ar}-\mathrm{H}), 12.96$ (s, 1H, SH). Calcd. for $\mathrm{C}_{15} \mathrm{H}_{12} \mathrm{ClN}_{3} \mathrm{OS}$ (301); (\%): C, 59.70; H, 4.01; N, 13.92; S, 10.62; found (\%): C, 59.58; H, 4.07, N, 14.00; S, 10.62.

5-Benzyl-4-(4-nitrophenyl)-4H-1,2,4-triazole-3-thiol (9d): Yield 63\%; m.p. 210-211 ${ }^{\circ} \mathrm{C}$; IR v ( $\left.\mathrm{cm}^{-1}\right)$ : 3354, $3291(\mathrm{~N}-\mathrm{H}), 2550(\mathrm{SH}), 1616(\mathrm{C}=\mathrm{N}), 1535,1260,1050,950(\mathrm{~N}-\mathrm{C}=\mathrm{S}$, amide I, II, III and IV bands); ${ }^{1} \mathrm{H}-\mathrm{NMR} \delta$ (ppm): 3.90 (s, 2H, $\left.\mathrm{CH}_{2}\right), 6.92-8.29$ (m, 9H, Ar-H), 14.01 (s, 1H, SH). Calcd. for $\mathrm{C}_{15} \mathrm{H}_{12} \mathrm{NO}_{2} \mathrm{~S}$ (312); (\%): C, 57.68; H, 3.87; N, 17.94; S, 10.27; found (\%): C, 57.58; H, 3.87, N, 18.00; S, 10.27 .

5-Benzyl-4-(4-methylphenyl)-4H-1,2,4-triazole-3-thiol (9e): Yield 71\%; m.p. 182-183 ${ }^{\circ} \mathrm{C}$; IR v $\left(\mathrm{cm}^{-1}\right)$ : 3358, $3290(\mathrm{~N}-\mathrm{H}), 2550(\mathrm{SH}), 1605(\mathrm{C}=\mathrm{N}), 1538,1262$, 1050, $950(\mathrm{~N}-\mathrm{C}=\mathrm{S}$, amide I, II, III and IV bands); ${ }^{1} \mathrm{H}-\mathrm{NMR} \delta(\mathrm{ppm}): 2.42\left(\mathrm{~s}, 3 \mathrm{H}, \mathrm{CH}_{3}\right), 3.81\left(\mathrm{~s}, 2 \mathrm{H}, \mathrm{CH}_{2}\right), 6.97-7.29(\mathrm{~m}, 9 \mathrm{H}, \mathrm{Ar}-\mathrm{H}), 12.91$ (s, 1H, SH). Calcd. for $\mathrm{C}_{16} \mathrm{H}_{15} \mathrm{~N}_{3} \mathrm{~S}(312)$; (\%): C, 68.30; H, 5.37; N, 14.93; S, 11.40; found (\%): C, 63.38; H, 5.37, N, $15.00 ; \mathrm{S}, 11.47$.

\section{General Procedure for the Preparation of Compounds 11a-d and 12a-d.}

A mixture of 7 (or 10) (0.01 mole) and the corresponding aryl aldehyde (0.01 mole) in ethanol (25 $\mathrm{mL})$ was treated with concentrated $\mathrm{HCl}(0.5 \mathrm{~mL})$ and refluxed for 2 hours. The reaction mixture on cooling was filtered and recrystallized from ethanol.

4-(Benzylideneamino)-5-furan-2-yl-4H-1,2,4-triazole-3-thiol (11a): Prepared from benzaldehyde, yield 59\%; m.p. 197-198 ${ }^{\circ} \mathrm{C}$; IR v (cm-1): 3329 (N-H), 2625 (SH), 1630 (C=N), 1238 (C-O-C), 1535, 1262, 1051, 952 (N-C=S, amide I, II, III and IV bands); ${ }^{1} \mathrm{H}-\mathrm{NMR} \delta$ (ppm): 6.57-7.23 (m, 3H, furan), 7.55-7.97 (m, 5H, Ar-H), 10.18 (s, 1H, N=CH), 13.19 (s, 1H, SH). Calcd. for $\mathrm{C}_{13} \mathrm{H}_{10} \mathrm{~N}_{4} \mathrm{OS}(270) ;(\%)$ : C, 57.76; H, 3.73; N, 20.73; S, 11.86; found (\%): C, 57.69; H, 3.71, N, 20.69; S, 11.89. 
5-furan-2-yl-4-[(4-methoxybenzylidene)amino]-4H-1,2,4-triazole-3-thiol (11b): Prepared from p-methoxybenzaldehyde, yield 53\%; m.p. 205-206 ${ }^{\circ} \mathrm{C}$; IR v $\left(\mathrm{cm}^{-1}\right): 3339(\mathrm{~N}-\mathrm{H}), 2525(\mathrm{SH}), 1630(\mathrm{C}=\mathrm{N})$, 1238 (C-O-C), 1545, 1262, 1050, 950 (N-C=S, amide I, II, III and IV bands); ${ }^{1} \mathrm{H}-\mathrm{NMR} \delta$ (ppm): 3.87 (s, $3 \mathrm{H}, \mathrm{OCH}_{3}$ ), 5.57-6.74 (m, 3H, furan), 7.07-7.97 (m, 4H, Ar-H), 9.18 (s, 1H, N=CH), 12.99 (s, 1H, SH). Calcd. for $\mathrm{C}_{14} \mathrm{H}_{12} \mathrm{~N}_{4} \mathrm{O}_{2} \mathrm{~S}(300)$; (\%): C, 55.99; H, 4.03; N, 18.65; S, 10.68; found (\%): C, 56.01; H, 3.99, N, 18.69; S, 10.67 .

4-[(4-Chlorobenzylidene)amino]-5-furan-2-yl-4H-1,2,4-triazole-3-thiol (11c): Prepared from p-chlorobenzaldehyde, yield 59\%; m.p. 225-226 ${ }^{\circ} \mathrm{C}$; IR v ( $\left.\mathrm{cm}^{-1}\right): 3359(\mathrm{~N}-\mathrm{H}), 2558(\mathrm{SH}), 1633(\mathrm{C}=\mathrm{N}), 1238$ (C-O-C), 1535, 1260, 1050, 950 (N-C=S, amide I, II, III and IV bands); ${ }^{1} \mathrm{H}-\mathrm{NMR} \delta$ (ppm): 5.71-6.84 (m, $3 \mathrm{H}$, furan), 7.06-7.98 (m, 4H, Ar-H), 9.98 (s, 1H, N=CH), 13.99 (s, 1H, SH). Calcd. for $\mathrm{C}_{13} \mathrm{H}_{9} \mathrm{ClN}_{4} \mathrm{OS}$ (304); (\%): C, 51.24; H, 2.98; N, 18.38; S, 10.52; found (\%): C, 51.19; H, 3.00, N, 18.41; S, 10.55.

4-[(3-Nitrobenzylidene)amino]-5-furan-2-yl-4H-1,2,4-triazole-3-thiol (11d): Prepared from m-nitrobenzaldehyde, yield 51\%; m.p. 165-166 ${ }^{\circ} \mathrm{C}$; IR v $\left(\mathrm{cm}^{-1}\right): 3369(\mathrm{~N}-\mathrm{H}), 2563(\mathrm{SH}), 1631(\mathrm{C}=\mathrm{N}), 1238$ (C-O-C), 1535, 1260, 1050, 950 (N-C=S, amide I, II, III and IV bands); ${ }^{1} \mathrm{H}-\mathrm{NMR} \delta$ (ppm): 2.40 (s, $3 \mathrm{H}$, $\mathrm{CH}_{3}$ ), 5.82-6.72 (m, 3H, furan), 7.40-8.71 (m, 4H, Ar-H), 10.08 (s, 1H, N=CH), 13.97 (s, 1H, SH). Calcd. for $\mathrm{C}_{13} \mathrm{H}_{9} \mathrm{~N}_{5} \mathrm{O}_{3} \mathrm{~S}(315)$; (\%): C, 49.52; H, 2.88; N, 22.21; S, 10.17; found (\%): C, 49.59; H, 3.00, N, 22.29; $\mathrm{S}, 10.25$.

5-Benzyl-4-(benzylideneamino)-4H-1,2,4-triazole-3-thiol (12a): Prepared from benzaldehyde, yield 43\%; m.p. 177-178 ${ }^{\circ} \mathrm{C}$; IR v ( $\left.\mathrm{cm}^{-1}\right): 3350(\mathrm{~N}-\mathrm{H}), 2558(\mathrm{SH}), 1610(\mathrm{C}=\mathrm{N}), 1535,1260,1050,950(\mathrm{~N}-\mathrm{C}=\mathrm{S}$, amide I, II, III and IV bands); ${ }^{1} \mathrm{H}-\mathrm{NMR} \delta$ (ppm): 4.18 (s, 2H, $\left.\mathrm{CH}_{2}\right), 7.28-7.90$ (m, 10H, Ar-H), 10.31 (s, $1 \mathrm{H}, \mathrm{N}=\mathrm{CH}), 12.99$ (s, 1H, SH). Calcd. for $\mathrm{C}_{16} \mathrm{H}_{14} \mathrm{~N}_{4} \mathrm{~S}$ (294); (\%): C, 65.28; H, 4.79; N, 19.03; S, 10.89; found (\%): C, 65.30; H, 4.81, N, 18.99.; S, 10.84 .

5-Benzyl-4-[(4-methoxybenzylidene)amino]-4H-1,2,4-triazole-3-thiol (12b): Prepared from p-methoxybenzaldehyde, yield 53\%; m.p. 191-192 ${ }^{\circ} \mathrm{C}$; IR v ( $\left.\mathrm{cm}^{-1}\right): 3354(\mathrm{~N}-\mathrm{H}), 2566(\mathrm{SH}), 1605(\mathrm{C}=\mathrm{N}), 1535$, 1260, 1050, 950 (N-C=S, amide I, II, III and IV bands); ${ }^{1} \mathrm{H}-\mathrm{NMR} \delta(\mathrm{ppm}): 4.16$ (s, 2H, $\left.\mathrm{CH}_{2}\right), 4.21$ (s, 3H, $\left.\mathrm{OCH}_{3}\right), 6.93-7.86(\mathrm{~m}, 9 \mathrm{H}, \mathrm{Ar}-\mathrm{H}), 10.02(\mathrm{~s}, 1 \mathrm{H}, \mathrm{N}=\mathrm{CH}), 12.79(\mathrm{~s}, 1 \mathrm{H}, \mathrm{SH})$. Calcd. for $\mathrm{C}_{17} \mathrm{H}_{16} \mathrm{~N}_{4} \mathrm{~S}(324)$; (\%): C, 62.94; H, 4.97; N, 17.27; S, 9.88; found (\%): C, 63.00; H, 4.96, N, 17.29.; S, 9.86.

5-Benzyl-4-[(4-chlorobenzylidene)amino]-4H-1,2,4-triazole-3-thiol (12c): Prepared from p-chlorobenzaldehyde, yield 58\%; m.p. 205-206 ${ }^{\circ} \mathrm{C}$; IR v $\left(\mathrm{cm}^{-1}\right): 3355(\mathrm{~N}-\mathrm{H}), 2561(\mathrm{SH}), 1625(\mathrm{C}=\mathrm{N}), 1533$, 1261, 1050, 950 (N-C=S, amide I, II, III and IV bands); ${ }^{1} \mathrm{H}-\mathrm{NMR} \delta$ (ppm): 4.17 (s, 2H, $\left.\mathrm{CH}_{2}\right), 7.27-7.96$ (m, 9H, Ar-H), 10.33 (s, 1H, N=CH), 13.79 (s, 1H, SH). Calcd. for $\mathrm{C}_{16} \mathrm{H}_{13} \mathrm{ClN}_{4} \mathrm{~S}$ (328); (\%): C, 58.44; H, 3.98; N, 17.04; S, 9.75; found (\%): C, 58.39; H, 3.96, N, 17.09.; S, 9.71. 
5-Benzyl-4-[(3-nitrobenzylidene)amino]-4H-1,2,4-triazole-3-thiol (12d): Prepared from m-nitrobenzaldehyde, yield 69\%; m.p. 181-182 ${ }^{\circ} \mathrm{C}$; IR v ( $\left.\mathrm{cm}^{-1}\right): 3359(\mathrm{~N}-\mathrm{H}), 2563(\mathrm{SH}), 1621(\mathrm{C}=\mathrm{N}), 1538$, 1260, 1050, 950 (N-C=S, amide I, II, III and IV bands); ${ }^{1} \mathrm{H}-\mathrm{NMR} \delta$ (ppm): 2.44 (s, 3H, $\left.\mathrm{CH}_{3}\right), 4.15$ (s, $2 \mathrm{H}$, $\mathrm{CH}_{2}$ ), 7.27-7.96 (m, 9H, Ar-H), 10.33 (s, 1H, N=CH), 13.91 (s, 1H, SH). Calcd. for $\mathrm{C}_{16} \mathrm{H}_{13} \mathrm{~N}_{5} \mathrm{~S}(339)$; (\%): C, 56.63; H, 3.86; N, 20.64; S, 9.45; found (\%): C, 56.69; H, 3.96, N, 20.69.; S, 9.41.

\section{References}

1. Unangst, P.C.; Shurum, G.P.; Connor, D.T.; Dyer, R.D.; Schrier, D.J.; J. Med. Chem. 1992, 35, 3691-3698.

2. Mullican, M.D.; Wilson, M.W.; Connor, D.T.; Kostlan, C.R.; Schrier, D.J.; Dyer, R.D.; J. Med. Chem. 1993, 36, 1090-1099.

3. Jones, D.H.; Slack, R.; Slack, R.; Squires, S.;Wooldridge, K.R.H.; J. Med. Chem. 1965, 8, 676-680.

4. Sughen, J.K.; Yoloye, T.; Pharm. Acta Helv. 1978, 58, 64-68.

5. Shams El-Dine, Sh.A.; Hazzaa, A.A.B. Pharmazie. 1974, 29, 761-768.

6. Misato, T.; Ko, K.; Honma, Y,; Konno, K.; Taniyama, E.; JP 77-25028 (A01N 9/12); [Chem. Abstr. 1977, 87, 147054a].

7. Cansız, A.; Servi, S.; Koparır, M.; Altıntaş, M.; Dı̆̆rak, M.; J. Chem. Soc. Pak. 2001, 23, 237-239.

8. Stillings, M.R.; Welbourn, A.P.; Walter, D.S.; J. Med. Chem. 1986, 29, 2280-2284.

9. Kane, J.M.; Dudley, M.W.; Sorensen, S.M.; Miller, F.P.; J. Med. Chem. 1988, 31, 1253-1258.

10. Porsolt, R.D.; Bertin, A.; Jalfre, M.; Arch. Int. Pharmacol. 1977, 229, 327-336.

11. Vamvakides, A.; Pharm. Fr. 1990, 48, 154-159.

Sample availability: Available from the authors.

(C) 2004 by MDPI (http:www.mdpi.org). Reproduction is permitted for noncommercial purposes. 